J. Appl. Numer. Optim. 3 (2021), No. 1, pp. 211-214

Available online at http://jano.biemdas.com

https://doi.org/10.23952/jano.3.2021.1.12

\title{
OPTIMAL CONTROL IN THE TRAFFIC FLOW: MULTI-LANE SIGNALIZED INTERSECTION
}

\author{
ILYA IOSLOVICH
}

Faculty of Civil and Environmental Engineering, Technion, Haifa 32000, Israel

\begin{abstract}
The continuous traffic flow model is modified and extended to find optimal control analytical solutions for oversaturated multi-phase and multi-lane signalized intersection. The considered objectives are minimal weighted total delay and maximal weighted throughput.
\end{abstract}

Keywords. Signalized intersection; Optimal control; Traffic flow.

\section{INTRODUCTION}

Recently, the optimal control for the signalized intersection was investigated in much researches; see, e.g., [1], [2], [3], and [4]. In this paper, we modify and extend the traffic flow model to find the optimal control analytical solution for oversaturated multi-phase and multilane signalized intersection. Both minimal delay and maximal throughput objectives are considered.

\section{PRELIMINARIES}

We consider a continuous model for multi-lane and muli-phase signalized intersection. The control is associated with green split for each phase $u_{j}$ of $m$ signal phases, where each phase has the minimal green split value $\underline{u}_{j}$. Values $a_{i}$ correspond to the demand rate in the lane $i$. Values $q_{i}$ represent the queue for the lane $i$. For each of $n$ lanes, the throughway velocity $d_{i}$ is known. The set $K_{i}$ contains all the signal phase indices $j$ that allow the movement for lane $i$. The set $L_{j}$ contains all the lanes indices $i$ that are actively moved during the green phase $j$. The optimization criterion is the weighted with weights $w_{i}$ total delay during time interval $T$. It is assumed that the intersection is oversaturated for all lanes for the time interval $[0, T]$.

E-mail address: agrilyaster@gmail.com.

Received September 12, 2020; September 21, 2020.

(C)2021 Journal of Applied and Numerical Optimization 
The model has the following form:

$$
\begin{aligned}
\frac{d q_{i}}{d t} & =a_{i}-d_{i}\left(\sum_{j \in K_{i}} u_{j}\right), \\
J & =\int_{0}^{T}\left(\sum_{i=1}^{n} w_{i} q_{i}\right) d t \rightarrow \min \\
u_{j} & \in U ; U=\left\{\sum_{j=1}^{m} u_{j}=1, \underline{u}_{j} \leq u_{j}\right\},
\end{aligned}
$$

It follows that

$$
u_{j} \leq \bar{u}_{j}=1-\sum_{k=1}^{m} \underline{u}_{k}+\underline{u}_{j}
$$

\section{OPTIMIZATION}

Utilizing Pontryagin maximum principle (PMP) according to [5], we consider the vector $p$ of costate variables $p_{i}(t)$ and form the Hamiltonian $H(p, q, u)$ as follows

$$
H=\sum_{i=1}^{n} p_{i}\left(a_{i}-d_{i} \sum_{j \in K_{i}} u_{j}\right)-\sum_{i=1}^{n} w_{i} q_{i} .
$$

The costate values must satisfy the differential equations

$$
\frac{d p_{i}}{d t}=-\frac{\partial H}{d q_{i}}=w_{i} .
$$

The final values of the queues $q_{i}(T)$ are free, which means that the transversality conditions must hold

$$
p_{i}(T)=0, i=1,2, \ldots, n .
$$

The control values $u_{i}$ have to be found from the maximization of the Hamiltonian over control variables with respect to their constraints. Thus, we have

$$
u_{j}=\arg \max _{u_{j} \in U} H(p, q, u) .
$$

As far as the lower bounds of $u_{j}$ are given, we just need to find

$$
\tilde{u}_{j}=u_{j}-\underline{u}_{j}
$$

Note that the coefficient for $u_{j}$ in $H$ is equal to

$$
S_{j}=-\sum_{i \in L_{j}} p_{i} d_{i}
$$

From equation (3.2) and transversality condition (3.3), we obtain that

$$
p_{i}(0)=-w_{i} T
$$

and

$$
p_{i}(t)=-w_{i} T+w_{i} t
$$


Substituting (3.7) into $S_{j}$, we get

$$
S_{j}(t)=(T-t) \sum_{i \in L_{j}} w_{i} d_{i}
$$

Now, the maximization of the Hamiltonian is reduced to the rather simple linear program for the variables $\tilde{u}_{j}$

$$
\begin{aligned}
\sum_{j=1}^{m} S_{j} \tilde{u}_{j} & \rightarrow \max , \\
\sum_{j=1}^{m} \tilde{u}_{j} & =1-\sum_{j=1}^{m} \underline{u}_{j}, \quad \tilde{u}_{j} \geq 0 .
\end{aligned}
$$

It follows that the optimal control is obtained from

$$
\begin{aligned}
j^{\text {opt }} & =\arg \max _{j} S_{j}, \\
\tilde{u}_{j^{o p t}} & =\overline{\tilde{u}}=1-\sum_{j=1}^{m} \underline{u}_{j}, \\
\tilde{u}_{j \neq j^{o p t}} & =0, \\
u_{j^{o p t}} & =\tilde{u}_{j^{o p t}}+\underline{u}_{j^{o p t}} \\
u_{j \neq j^{o p t}} & =\underline{u}_{j} .
\end{aligned}
$$

The correspondent dual problem for Lagrange multiplier $\lambda$ has the form

$$
\begin{aligned}
& I=\lambda\left(1-\sum_{j=1}^{m} \underline{u}_{j}\right) \rightarrow \min \\
& \lambda \geq S_{j}, \lambda \geq 0 .
\end{aligned}
$$

According to the KKT conditions, we have

$$
I-J \geq 0 .
$$

This is a so-called "dual gap". The solution of the dual problem is

$$
\lambda=S_{j^{o p t}}
$$

Thus we see that

$$
I-J=0,
$$

and the solution of the linear problem is optimal.

It is clear that the value of the index $j^{o p t}$ is not changed with time because the coefficient $(T-t)$ is always positive. This concludes the analytical solution of the optimal control problem.

\section{Maximal Throughrut Solution}

In the case of the maximal weighted throughput the objective has form

$$
J=\sum_{i=1}^{n} w_{i} q_{i}(T) \rightarrow \min
$$


i.e., here it is minimization of the function of the final states $F(q(T))$. In this case, the Hamiltonian does not contain terms with state variables and thus the costate equations are

$$
\frac{d p_{i}}{d t}=\frac{\partial H}{\partial q_{i}}=0, i=1,2, \ldots, n .
$$

Accordingly, the costates $p_{i}$ are constant. From the transversality conditions, we have

$$
p_{i}(T)=-\operatorname{grad}\left(F(q(T))=-w_{i} .\right.
$$

It follows that $p_{i}(t)=w_{i}$, and that the coefficients of controls $u_{j}$ in the Hamiltonian are

$$
S_{j}=\sum_{i \in L_{j}} w_{i} d_{i}
$$

i.e., they are the same as for the case of weighted minimal delay - just without common positive coefficient $(T-t)$. This means that the optimal solution is the same.

\section{CONCLUSiON}

The optimal control for the multi-lane signalized intersection was fully investigated and the analytical solution was presented. The solution is somehow similar to the known solution of the Continuous Knapsack Problem (CKP) [6].

\section{REFERENCES}

[1] D. Gazis, Optimum control of a system of oversaturated intersections, Oper. Res. 12 (1964), 815-831.

[2] J. Haddad, B.D. Schutter, D. Mahalel, et al., Optimal steady-state control for isolated traffic intersections, IEEE Trans. Auto. Control 55 (2010), 2612-2617.

[3] I. Ioslovich, J. Haddad, P.O. Gutman, et al., Optimal traffic control synthesis for an isolated intersection, Control Engineering Practice 19 (2011), 900-911.

[4] I. Ioslovich, J. Haddad, P.O. Gutman, et al., Design of optimal traffic flow control at intersection with regard for queue length constraints, Automation and Remote Control 72 (2011), 1833-1840.

[5] L. Pontryagin, V. Boltyanskii, R. Gamkrelidze, et al., The mathematical theory of optimal processes, WileyInterscience, NY, 1962.

[6] I. Ioslovich, Robust reduction of a class of large-scale linear programs, SIAM J Optim. 12 (2001), 262-282. 